\title{
Membrane immunoglobulin expressed by retroviral vector gene transfer mimics partial function of the B-cell receptor in vivo
}

\author{
Jing Lu, Feng Chen, Zhen Xu, Lingling Zhang, Peng Xu, Depei Liu* \\ \& Chihchuan Liang \\ State key Laboratory of Medical Molecular Biology, Department of Biochemistry \& Molecular Biology, Institute of Basic Medical Sciences, \\ Chinese Academy of Medical Sciences and Peking Union Medical College, Beijing 100005, China
}

Received July 16, 2015; accepted August 12, 2015; published online October 16, 2015

\begin{abstract}
Activation of B-cells is initiated by the ligation of B-cell receptors by its cognate antigen, inducing a series of signal cascades. Understanding the molecular mechanisms of these important events is a crucial goal for immunologists. Chimeric B cell receptors provide a powerful tool for analysis of B-cell signal function. However, this method can only be used in tool cells, but cannot be used for in vivo study. Here, we constructed a retroviral vector to encode both heavy chains and light chains of a membrane immunoglobulin, and expressed them in primary B-cells using retroviral gene transfer. Our results demonstrate that the membrane immunoglobulin expressed by retroviral vectors transfer can initiate B-cell receptor-mediated signaling, resulting in the phosphorylation of Syk and Erk1/2 proteins. The results showed that B-cells expressing membrane immunoglobulin can make proliferative responses to cognate antigen both in vitro and in vivo. Therefore, we provide a methodology for rapidly analyzing the downstream signals of B-cell receptors both in vitro and in vivo, which could expedite the identification of proteins involved in B-cell function.
\end{abstract}

BCR, membrane immunoglobulin, retroviral vector

Citation: $\quad$ Lu, J., Chen, F., Xu, Z., Zhang, L., Xu, P., Liu, D., and Liang, C. (2016). Membrane immunoglobulin expressed by retroviral vector gene transfer mimics partial function of the B-cell receptor in vivo. Sci China Life Sci 59: 49-58, doi: 10.1007/s11427-015-4931-3

\section{INTRODUCTION}

Various signals act via the B-cell receptor (BCR) to induce B-cell activation, clonal expansion, differentiation, apoptosis, and regulate B-cell-related immune responses (Niiro and Clark, 2002). Activation of B-cells is initiated by the ligation of the BCR by its cognate antigen, and this opens the gate for a series of signal cascades (Kurosaki, 2011; Reth and Wienands, 1997), finally resulting in the production of antibodies against pathogenic invaders. Understanding the detailed molecular mechanisms of these important events is a crucial goal for immunologists.

Chimeric receptors composed of immunoglobulins (Igs)

*Corresponding author (email: liudp@pumc.edu.cn) and individual signaling components provide a powerful tool for analysis of B-cell signal function. However, this method can only be used in tool cells such as A20 cells or in the myeloma cell line J558L $\mu$ m (Flaswinkel and Reth, 1994; Sanchez et al, 1993). A20 is a BALB/c lymphoma, which has been used extensively for in vitro studies of antigen processing (Glimcher et al, 1982). J558L $\mu \mathrm{m}$ cells do not carry the cluster of differentiation 45 (CD45) surface marker and do not undergo any calcium response (Justemen et al, 1991). Therefore, these cells can only be used in vitro for the study of BCR-mediated signaling and cannot be used for in vivo study. Although the use of in vitro systems has provided valuable information, some complications such as activation and expansion are best analyzed in vivo after cognate antigen activation. Additionally, only one or two 
antigen-specific BCRs are typically studied because no convenient methods exist to analyze the complex chain of events downstream of BCR. There is a need to develop new methodology that can facilitate the study of BCR-related events both in vitro and in vivo.

BCRs located on mature B-cells contain membrane immunoglobulin (mIg) — an antigen-binding subunit that consists of two heavy chains ( $\operatorname{IgH})$ and two light chains ( $\operatorname{IgL})$ of immunoglobulin - and a signaling subunit, which is a disulfide-linked heterodimer of the Ig- $\alpha$ (CD79a) and Ig- $\beta$ (CD79b) proteins. In the BCR complex, mIg alone determines antigen specificity. Therefore, if we construct a vector harboring the cDNA of both IgHs and IgLs, the mIg expressed by the vector may interact with the transmembrane domains of the Ig- $\alpha /$ Ig- $\beta$ (CD79) heterodimer.

In this study, we constructed a retroviral vector containing the cDNA of both the IgHs and IgLs of a mIg and expressed this $\mathrm{mIg}$ in spleen B-cells using retroviral gene transfer. We found that the $\mathrm{mIg}$ expressed by a retroviral vector gene transfer can make proliferative responses to cognate antigen both in vitro and in vivo. Our study provides a method to analyze the function of antigen-specific BCRs both in vitro and in vivo.

\section{RESULTS}

\section{Construction of retroviral vector carrying mIg- ex- pressing cassettes}

Multiple internal promoters can be included in a retroviral vector. It is possible to express at least three different cDNAs, each from its own promoter (Overell et al, 1988). Based on this knowledge, we chose two promoters to control the expression of $\operatorname{IgH}$ and $\operatorname{IgL}$. Before retroviral vector construction, we used a reporter assay to detect the activity of several promoters (Figure 1). According to the luciferase assay results, we chose the PGK and CMV promoters for the flowing retroviral vector construction based on two considerations. On the one hand, packaging efficacy inversely correlates with the length of the retroviral construct, which limits the titers that can be achieved with this approach. Although the hEF1a and $\mathrm{m} \beta$-actin promoters scored strongest in the luciferase assay, they were too long to be suitable for retroviral vector packaging. On the other hand, the high activity of internal promoters may hinder 5'-LTR-driven EGFP expression (details in discussion). Compared with hEF1a and $\mathrm{m} \beta$-actin promoters, relative shorter length and modest activity of the PGK and CMV promoters make them better choices for the following retroviral vector construction.

We carried out the retroviral vector construction as follows. First, we cloned the EGFP gene into a mouse stem cell virus (MSCV)-based retroviral vector to facilitate the identification of marked cells. The expression of the EGFP

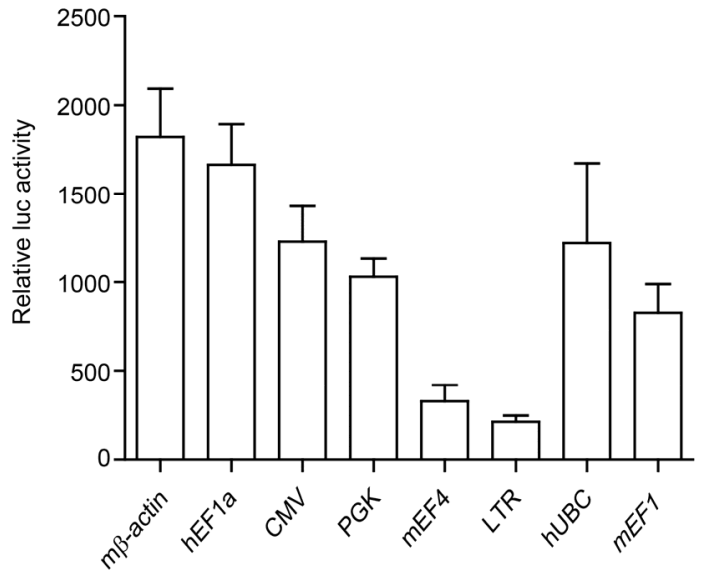

Figure 1 Activity of eight promoters analyzed by reporter assay. Activity of eight promoters was detected using dual-luciferase reporter assay in NIH3T3 cell line. The m $\beta$-actin, hEF1 $\alpha$, CMV, PGK, mEF4, LTR, hUBC and $\mathrm{mEF} 1 \alpha$ promoters were cloned into pGL3-basic vectors and sequenced to assure fidelity. NIH3T3 cell line was transfected with these eight vectors. Luciferase activity was assayed $48 \mathrm{~h}$ after transfection using the Promega Luciferase Assay System according to the manufacturer's instructions. Luciferase activity was normalized against transfection efficiency by using a pRL-CMV reporter vector (Promega) as an internal control. Results were expressed as the ratio of firefly luciferase to renilla luciferase activity. Error bars represent standard deviations as calculated from triplicates. $\mathrm{m} \beta$-actin, mouse $\beta$-actin; hEF1 $\alpha$, human elongation factor $1 \alpha$; mEF4, mouse elongation factor 4 ; LTR, long terminal repeat; hUBC, human ubiquitin $\mathrm{C} ; \mathrm{mEF} 1 \alpha$, mouse elongation factor $1 \alpha$.

gene is controlled by a 5'LTR promoter (Figure 2A). Second, $\operatorname{IgG} 1$ heavy chain (IgH) and light chain (IgL) expression cassette elements were cloned into the retroviral vector mentioned above (Figure 2B). Expressions of IgH and IgL were controlled by the CMV and PGK promoters, respectively. According to the manufacturer's instructions of the retroviral vector, the transcribed mRNAs share the poly-A site in 3'LTR. All the cDNAs of EGFP, $\operatorname{IgH}$ and $\operatorname{IgL}$ harbor a termination code for their own translation termination. The resulting vector encodes not only the detection marker of EGFP but also the mIg.

\section{Both mIg and EGFP encoded by retroviral vector can be simultaneously expressed in primary B-cells}

In theory, mIg can be expressed by retroviral vector pMEIg, and specifically bind the cognate antigen. To validate those assumptions, primary B-cells were infected with retroviral vector pMEIg and, after $48 \mathrm{~h}$, stained with labeled cognate antigen then analyzed by a fluorescence microscope. As expected, the expression of EGFP can be detected in pMEIg transfectant (green, Figure 3A). Expression of mIg can also be detected by labeled cognate antigen in the same cell (red, Figure 3A). To validate further the simultaneous expression of EGFP with $\mathrm{mIg}$, primary B-cells infected with a retroviral vector expressing $\mathrm{mIg}$ (pMEIg) were analyzed by flow cytometry. Retroviral vector pMSCV-neo which expresses neither EGFP nor mIg was used as a negative control. In- 
fection efficiency was estimated by flow cytometric evaluation of the percentage of EGFP-expressing B cells. Infection efficiency by retroviral vector pMEIg and pMEGFP were $9.5 \%(9.5 \% \pm 0.6 \%, n=6)$ and $11.4 \%(11.4 \% \pm 0.8 \%, n=6)$ respectively (Figure 3B). We also found that approximately $40 \%$ of EGFP-expressing B-cells express $\mathrm{mIg}$ when infected by retroviral vector pMEIg (Figure 3B). No expression of $\mathrm{mIg}$ was detected in primary B-cells infected with the control retroviral vector (pMSCV-neo), which also lacked EGFP expression (Figure 3B). No expression of mIg was detected in primary B-cells infected with the control retroviral vectors (pMSCV-neo and pMEGFP), which lacked
mIg expression (Figure 3B). These results show that both $\mathrm{mIg}$ and EGFP encoded by retroviral vector pMEIg can be simultaneously expressed in primary B-cells.

\section{Cognate antigen binding can cause phosphorylation of Syk and Erk1/2 proteins}

In addition to the antigen-binding $\mathrm{mIg}, \mathrm{BCR}$ contains $\mathrm{Ig}$ alpha (Ig- $\alpha)$ and Ig beta (Ig- $\beta)$. In our design, a retroviral vector expressing $\mathrm{mIg}$ was associated with the disulfidelinked heterodimer Ig- $\alpha / \operatorname{Ig}-\beta$, which are the IgG1 subunits responsible for signal transduction. In order to examine the

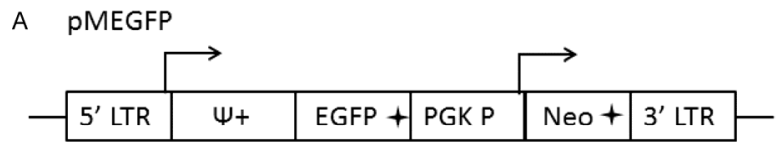

B pMEIg

\begin{tabular}{|c|c|c|c|c|c|c|c|c|}
\hline $5^{\prime}$ LTR & $\Psi+$ & EGFP + & PGK P & $\mathrm{H}$ chain & + & CMVP & L chain $t$ & 3' LTR \\
\hline
\end{tabular}

Figure 2 Structure of retroviral vectors pMEGFP and pMEIg. Expression of the EGFP gene is controlled by the 5'LTR promoter. Expressions of IgH and IgL are controlled by the CMV and PGK promoters, respectively. Arrows represent transcription initiation sites for each of the three promoters. The transcribed mRNAs share the poly-A site in 3'LTR. 5'LTR, 5' long terminal repeat; $\psi+$, extended packaging signal; EGFP, enhanced green fluorescent protein gene; PKG P, murine phosphoglycerate kinase promoter; H-chain, the cDNA of heavy chain; CMV P, Human cytomegalovirus immediate early promoter; L-chain, the cDNA of light chain; 3'LTR, 3' long terminal repeat (harboring poly-A site); Cross stars represent translation termination sites.

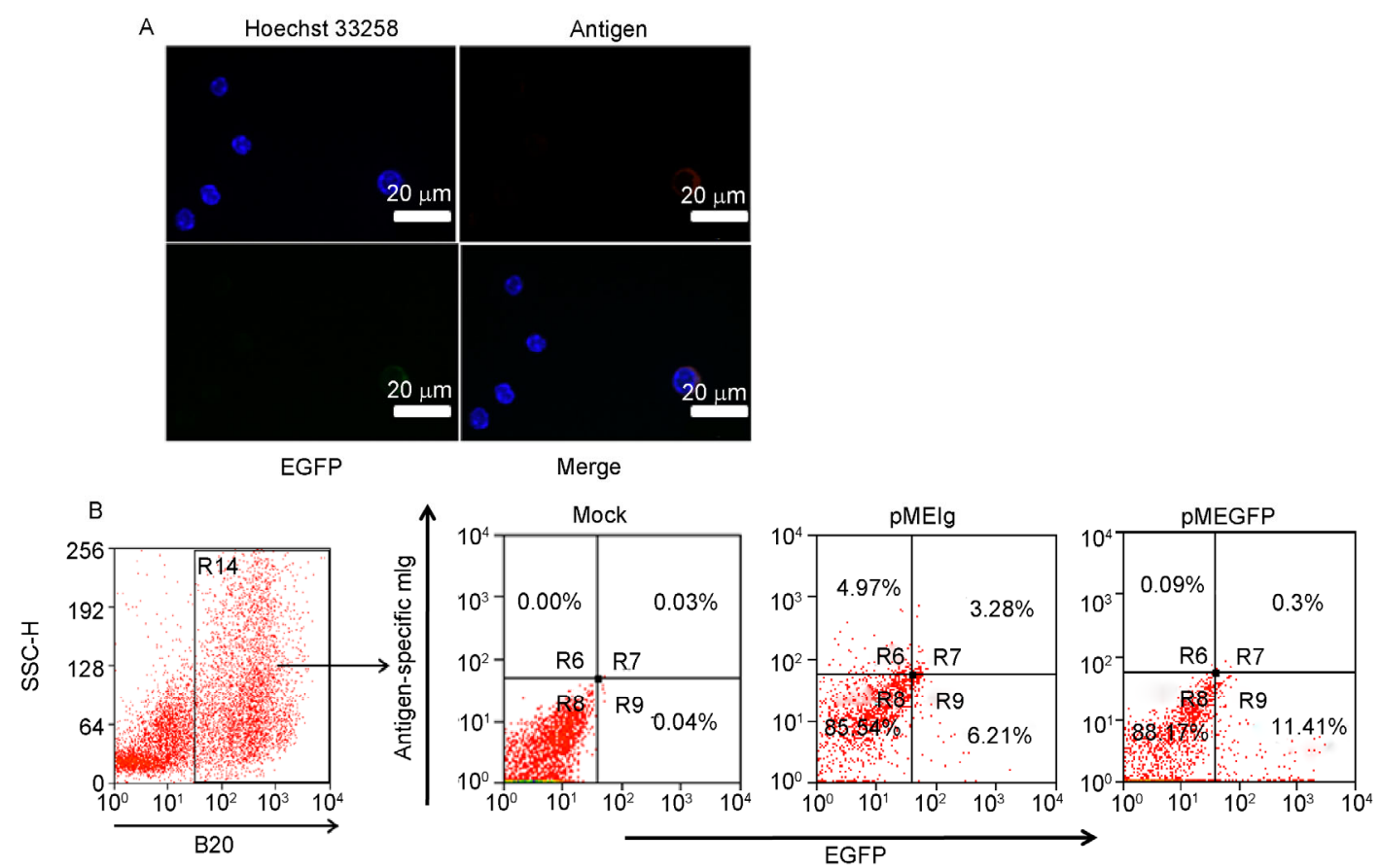

Figure 3 Both $m I g$ and EGFP encoded by retroviral vector can be simultaneously expressed in primary B-cell. A, Primary mouse splenocytes were infected with mIg-expressing-retroviral vector pMEIg and $48 \mathrm{~h}$ later stained by labeled cognate antigen (red) and the nuclei were stained with Hoechst33258 (blue), cells were photographed under a fluorescence microscope. Images were taken at $60 \times$ magnification. The scale bar represents $20 \mu \mathrm{m}$ in length. B, Primary mouse splenocytes were infected with pMSCV-neo (indicated as Mock) and mIg-expressing-retroviral vector (indicated as pMEIg) or non-mIg-expressing-retroviral vector (indicated as pMEGFP) and $48 \mathrm{~h}$ later the expression of $m I g$ and $E G F P$ were detected by flow cytometry. Percentage of $\mathrm{mIg}$ and $E G F P$ are indicated. All histograms are gated on $\mathrm{B} 220^{+}$cells (left, box gates). Representative data of three experiments are shown. 
initiation of BCR-mediated signaling, primary B-cells infected by the retroviral vector were stimulated by cognate antigen or were left unstimulated. The phosphorylation levels of Erk1/2 and Syk were analyzed by BD Phosflow. Infected primary B-cells were gated on $\mathrm{B} 220^{+} \mathrm{EGFP}^{+}$for flow cytometry analysis (Figure 4A). In the B-cells infected by mIg-expressing retroviral vector pMEIg (Figure 4B, left), Erk1/2 phosphorylation level is increased after cognate antigen stimulation (green line) compared with the unstimulated state (red line). To further exclude the possibility that non-specific increases in Erk1/2 phosphorylation levels could have been triggered by the antigens themselves, Bcells were also infected by a non-mIg-expressing control vector (pMEGFP). The results showed no significant increase in phosphorylation level of Erk1/2 after antigen stimulation (Figure 4B, middle). This result excludes the non-specific effect of the antigen. Similar results were also obtained for the Syk phosphorylation level (Figure 4C, right and middle). These results show that mIg expressed by retroviral vectors can initiate BCR-mediated signaling transduction.

\section{B-cells harboring vector-expressed mIg generate prolif- erative responses to cognate antigen in vitro}

After validating that mIg expressed by retroviral vectors can initiate BCR-mediated signaling transduction, we wanted to know that whether the B-cells harboring vector-expressed mIg could make proliferative responses to cognate antigen. We first used the murine primary cells to confirm that the cells can get well proliferated. The results showed that approximately $20 \%$ murine primary cells can be proliferated with the stimulation of LPS (Figure 5A). This result demonstrates that the murine primary cells can be used in this assay. Then we tested the proliferative response by EdU staining in the presence of CD40L, which generated "signal two" of the "two-signal theory" of lymphocyte recognition and activation. After infection with mIg-expressing vector pMEIg, the cells were either treated with cognate antigen or PBS or left untreated as a control. Because only a small proportion of B-cells were infected by the retroviral vector, EGFP was used as a marker of the infected B-cells for evaluation of the proliferation of B-cells that express $\mathrm{mIg}$
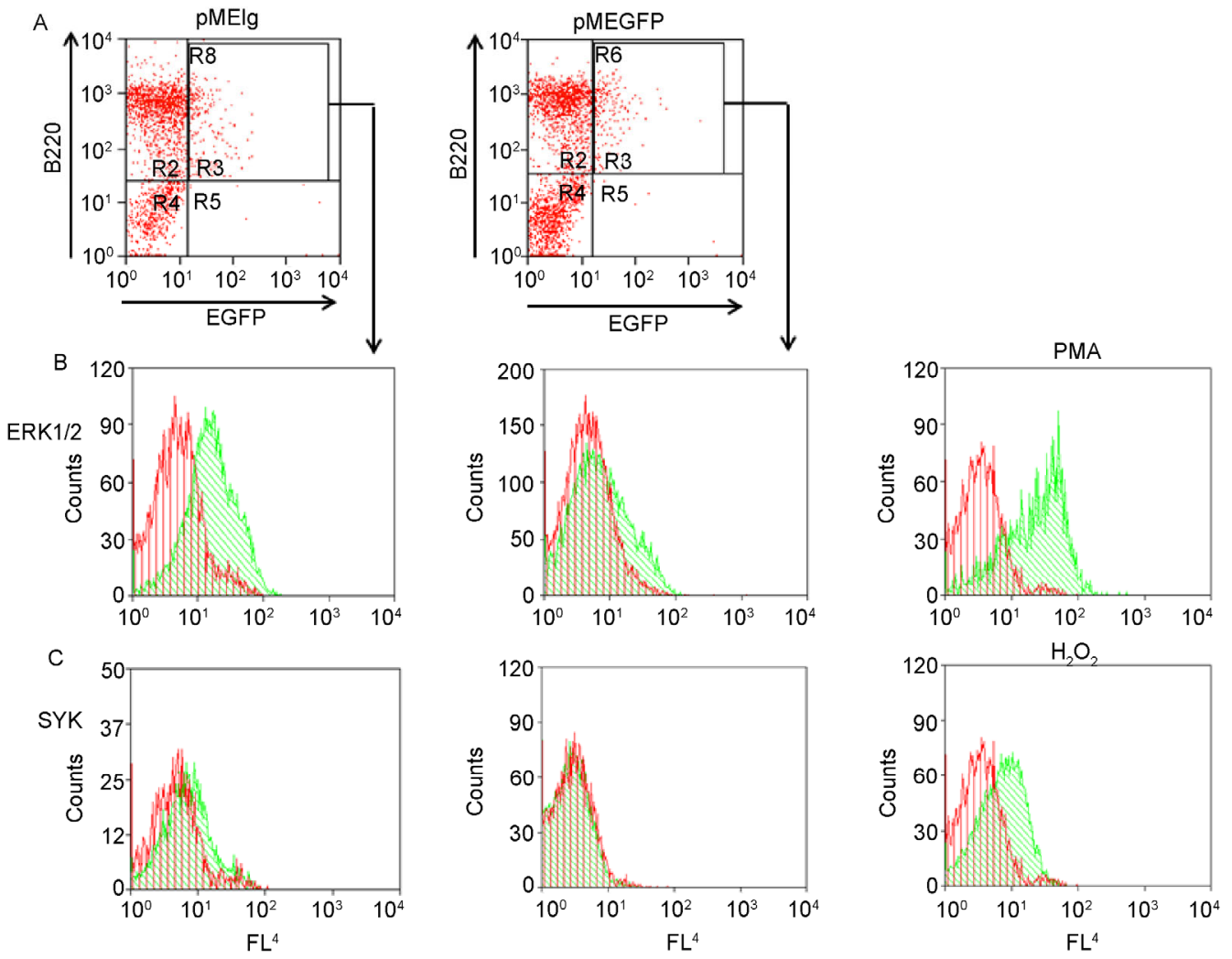

Figure 4 Cognate antigen binding can cause phosphorylation of Syk and Erk1/2 proteins. A, Retroviral vector infected primary mouse B-cells were gated on $\mathrm{B} 220^{+} \mathrm{EGFP}^{+}$for further intracellular phosphospecific flow cytometry analysis. B, The phosphorylation of Erk1/2 protein of primary-B-cells infected with mIg-expressing-retroviral vector pMEIg (left) and non-mIg-expressing-retroviral vector pMEGFP (middle) were detected by flow cytometry. Overlay histogram represents pre-incubated with cognate antigen (green line) and without cognate antigen (red line). PMA-treated cells were used as a positive control of Erk1/2 protein phosphorylation (right). C, The phosphorylation of Syk protein of primary-B-cells infected with mIg-expressing-retroviral vector pMEIg (left) and non-mIg-expressing-retroviral vector pMEGFP (middle) were detected by flow cytometry. Overlay histogram represents pre-incubated with cognate antigen (green line) and without cognate antigen (red line). $\mathrm{H}_{2} \mathrm{O}_{2}$-treated cells were used as a positive control of Syk protein phosphorylation (right). Results are representative of three independent experiments. 
(Figure 5B). The proliferation rate $\left(\mathrm{EdU}^{+} \mathrm{EGFP}^{+}\right.$cells/ $\left(\mathrm{EdU}^{+} \mathrm{EGFP}^{+}\right.$cells+EdU $\mathrm{EGFP}^{+}$cells $)$) was used to evaluate the proliferation induced by cognate antigen. The results show that the antigen+CD40L group had a relatively higher proliferation rate compared with both the CD40L group and control group (Figure 5B, upper, ${ }^{*}, P<0.05$ ). To further exclude the possibility of a non-specific increase by antigen, B-cells were also infected by a non-mIg-expressing vector. Results indicated no increase in proliferation in the antigen+ CD40L group compared with both the CD40L group and the control group (Figure $5 \mathrm{~B}$, lower, $P>0.05$ ). These results demonstrate the B-cells harboring vector-expressed mIg can make proliferative responses to cognate antigen.

We have previously proved that both mIg and EGFP encoded by a retroviral vector can be expressed simultaneously in a primary B-cell, we wanted to know whether
EGFP alone could be used as a marker for the detection of proliferative response. We measured the proliferative response without EdU staining (Figure 6A). The results show that the antigen+CD40L group mounted a relatively higher frequency of EGFP-positive cells compared to the CD40L group and control group (Figure 6B, left, **, $P<0.005$ ). To exclude further the possibility that non-specific increase expression levels could have been triggered by the antigen itself, B-cells were also infected by a non-mIg-expressing vector. As expected, no increase in EGFP-positive cells in the antigen+CD40L group was observed relative to the CD40L group and control group (Figure 6B, right, $P>0.05$ ). These data demonstrate that $\mathrm{B}$-cells harboring a mIgexpressing vector can make proliferative responses to cognate antigen. These results are in agreement with the EdU staining results. We can conclude that EGFP alone can be

A
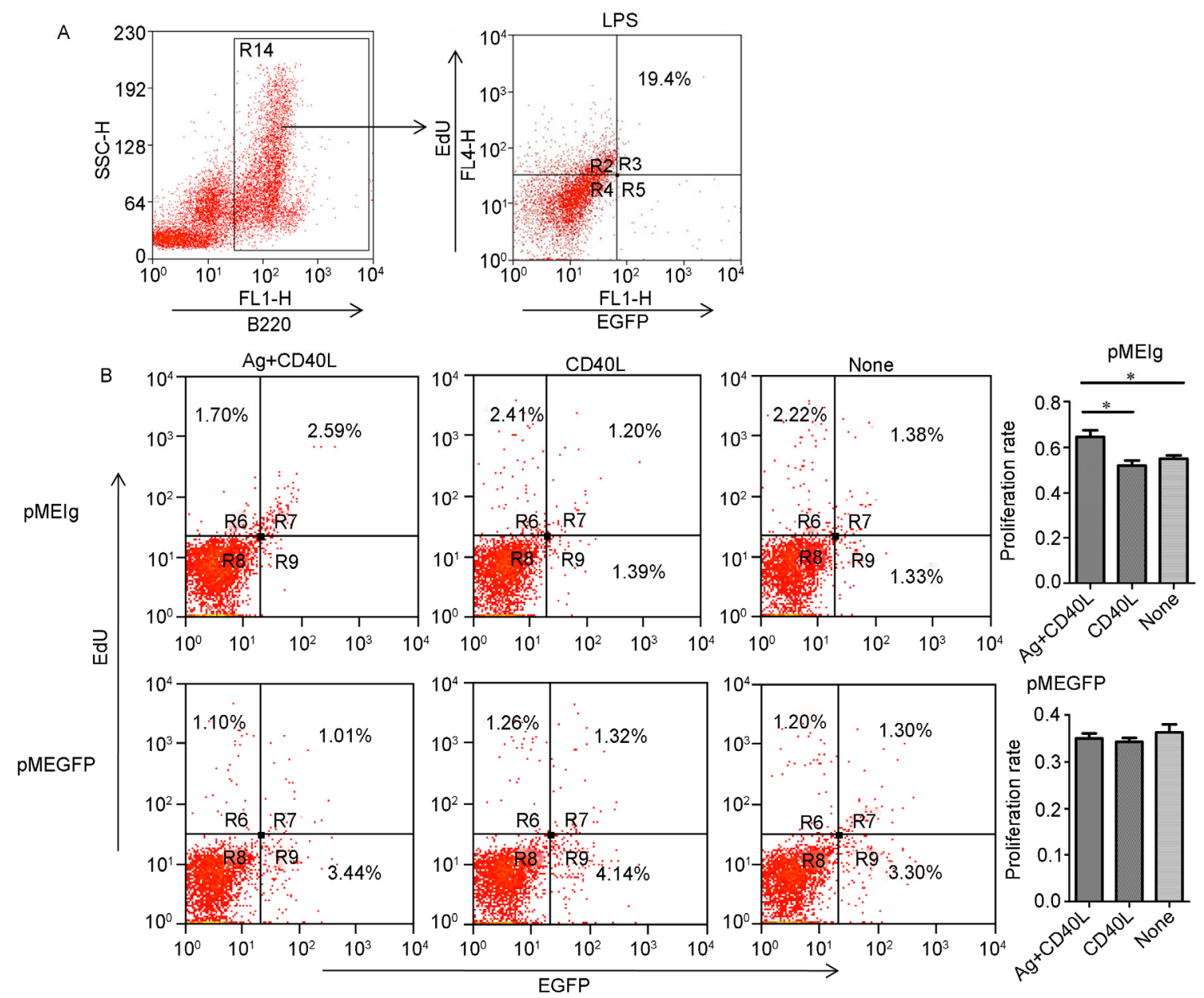

Figure 5 B-cells harboring vector-expressed mIg generate proliferative responses to cognate antigen in vitro. A, The proliferative response was detected by EdU staining using the murine primary cells with the stimulation of LPS. Results are representative of three independent experiments. B, The proliferative response was detected by EdU staining at the presence of CD40 ligand. After infection with mIg-expressing vector pMEIg, primary B-cells were treated with cognate antigen and CD40 Ligand, or treated with CD40 Ligand only, or left untreated indicated as control. Because a little of B-cells were infected by the mIg-expressing retroviral vector, EGFP was used as a marker of the infected B-cells for accurate evaluation the proliferation of mIg expressing B-cells. Data shown are representative of three independent experiments. The proliferation rate (EdU $\mathrm{EGFP}^{+}$cells/(EdU ${ }^{+} \mathrm{EGFP}^{+}$cells+EdUEGFP ${ }^{+}$cells)) was used to evaluate the proliferation induced by antigen. Results are representative of three independent experiments. The statistical significance is determined by one-way ANOVA. $P$-value is indicated $(*, P<0.05)$. 
the detecting marker for the proliferation assay.

\section{B-cells harboring vector-expressed $\mathrm{mIg}$ made prolifera- tive responses to cognate antigen in vivo}

Signal transduction mediated by vector-expressed $\mathrm{mIg}$ was tested further in vivo. Splenocytes infected with retroviral vector pMEIg, which encode both EGFP and mIg genes, were adoptively transferred into SCID mouse recipients. Splenocytes infected with retroviral vector pMEGFP, which encodes only EGFP were used as a negative control. The recipients were then divided into two groups. One group was challenged with cognate antigen on day one after cell transfer. As a control, the other group was treated with PBS. After adoptive transfer, the splenocytes of recipients were separated and the frequency of EGFP-positive B-cells was detected by flow cytometry on day 18 and day 28 (Figure 7A). Results show that the frequency of EGFP positive B-cells in the antigen-challenged group is significantly higher than that in the PBS-treated group on day 18 (Figure $7 \mathrm{~B}, * * *, P<0.001)$. Similar results were observed on day 28. There is no difference of proliferation between the antigen
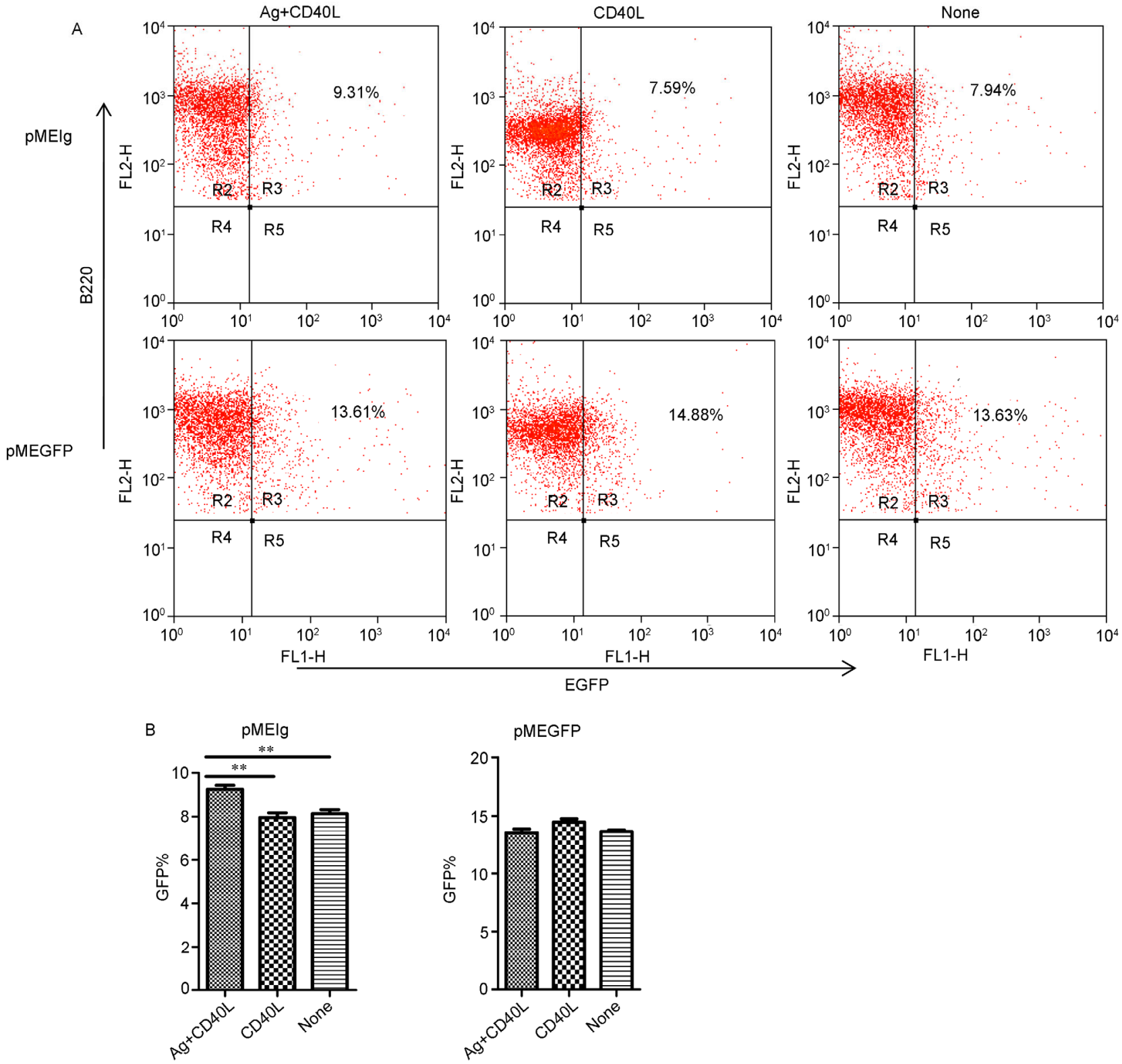

Figure 6 EGFP alone can be used as a marker for the detection of proliferative response. A, Retroviral vector pMEIg infected B-cells expressing EGFP and antigen-specific mIg were cultured for $48 \mathrm{~h}$ in the presence of antigen and CD40L, or in the presence of CD40L only, or left untreated indicated as none. Flow cytometry detected the frequency of EGFP positive cells. Retroviral vector pMEIg infected B-cells was used as a negative control. Data shown are representative of three independent experiments. B, The increased frequency of EGFP positive cells was used to evaluate the proliferation induced by antigen. The statistical significance is determined by one-way ANOVA. $P$-value is indicated $(* *, P<0.005)$. 
challenged group and un-challenged group when using control vector pMEGFP on day 18 and day 28 (Figure 7C and $\mathrm{D}, P>0.05)$.

\section{DISCUSSION}

In this study, we demonstrate that the membrane immunoglobulin expressed by retroviral vectors can initiate B-cell receptor-mediated signaling, inducing the downstream signals of B-cell receptors. To our knowledge, this is the first report revealing that B-cells harboring vector-expressed $\mathrm{mIg}$ make proliferative responses to cognate antigen both in vitro and in vivo.

This approach has two key advantages. First, our approach is substantially faster than transgenic technology, with retroviral vector construction and B-cell infection ready to analyze after 3 weeks. Second, although we examined only one BCR construct in this study, it is possible in theory that multiple BCRs can be examined simultaneously. This methodology is suitable for the study of analyzing the downstream signals and some processes of B-cell receptor, such as signal cascades, activation and expansion both in vitro and in vivo. However, we suggest that this methodology is not suitable for some studies, such as somatic hypermutation (SHM) and class switch recombination (CSR)
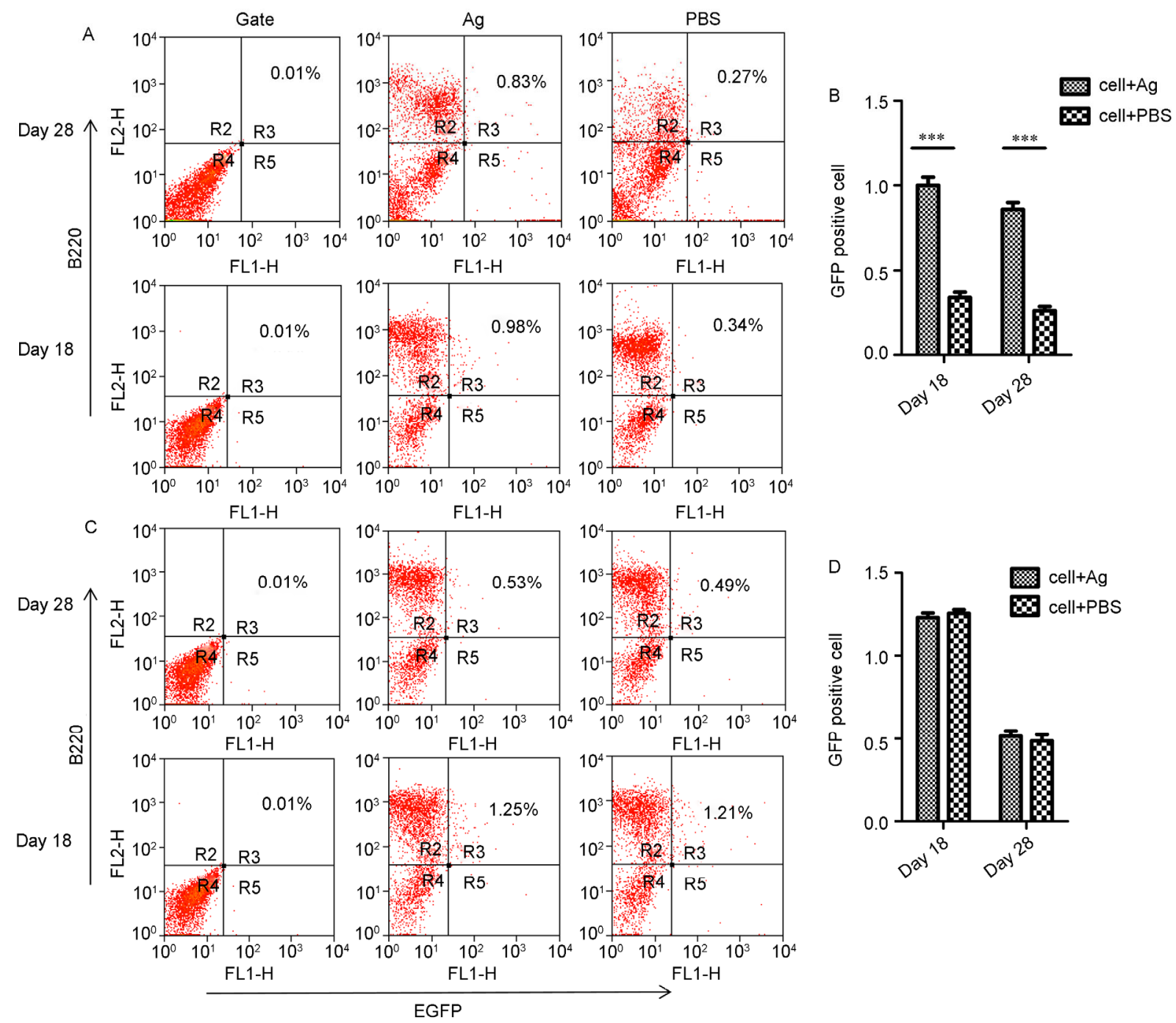

Figure 7 B-cells harboring vector-expressed $\mathrm{mIg}$ made proliferative responses to cognate antigen in vivo. A, Splenocytes $\left(3 \times 10^{7}\right)$ infected by a mIg-expressing retroviral vector pMEIg were transferred intravenously into SCID Beige mice. The recipients were then divided into two groups $(n=4)$. One group was challenged with cognate antigen. As a control, the other group was treated with PBS ( $n=4)$. Flow cytometry analysis was performed to determine the frequency of B220 $\mathrm{EGFP}^{+}$cells in Antigen-treated group (indicated as Ag) and PBS-treated (indicated as PBS) group on day 18 and 28 . B, The frequency of EGFP positive B-cells was used to evaluate the proliferation induced by antigen. The statistical significance is determined by two-way ANOVA. $P$-value is indicated $(* * *, P<0.001)$. C, Splenocytes $\left(3 \times 10^{7}\right)$ infected by a retroviral vector pMEGFP were transferred intravenously into SCID Beige mice. Flow cytometry analysis was performed to determine the frequency of $\mathrm{B}_{22} 20^{+} \mathrm{EGFP}^{+}$cells in Antigen-treated group (indicated as Ag) and PBS-treated group (indicated as PBS) on day 18 and 28. D, The frequency of EGFP positive B-cells was used to evaluate the proliferation induced by antigen. The statistical significance is determined by two-way ANOVA. $P$-value is indicated $(P>0.05)$. 
studies. The switch region is not contained in our mIgexpressing vector. Switch regions are a target for SHM, and sustain as high a frequency of mutation as the $\mathrm{V}$ region. Knockout mice with a partial deletion of $\mathrm{S} \mu$ tandem repeats have revealed that the deletion decreased CSR (Luby et al, 2001; Schrader et al, 2007). When the entire $4.6 \mathrm{~kb}$ region containing $\mathrm{S} \mu$ tandem repeat sequences was deleted, both SHM and CSR were ablated (Khamlichi et al, 2004). Therefore, CSR, SHM and affinity maturation will not take place in our mIg-expressing vector. Based on the considerations mentioned above, our methodology can be used to analyze the process of activation and expansion of B-cells, but may not be well-suited for the study of SHM and CSR.

We chose dual open reading frame version based on two promoters for the expression of $\mathrm{mIg}$, instead of a single open reading frame version based on $2 \mathrm{~A}$ sequence, which are widely used for expression multi-gene in a single vector. Our approach avoids the effect of the $2 \mathrm{~A}$ tag, which remains attached to the N-terminal cistron (Szymczak et al, 2004; Szymczak-Workman et al, 2012). This may affect signal processing or contribute to the antigenicity of the mIg expressed by the retroviral vector. However, the use of internal promoter elements may results in a small numbers of EGFP-expressing B cells simultaneously expressing introduced membrane IgG1 (Figure 3B). Because the internal promoters and the LTR-driven expression share the same transcription site (Poly A) at the 3'-end of retroviral vector pMEIg (Figure 2B), the LTR driven transcription may be influenced by the internal promoters. The other reason is the lower driven-activity of LTR promoter compared with the internal promoters (Figure 1, LTR vs. CMV and PGK). The use of internal promoters may also reduce the titer of our retroviral vector because retroviral RNA is also transcribed by the LTR promoter. Based on these considerations, the use of internal promoter with lower activity may result in less interference with LTR-driven transcription and thus increase expression of EGFP. The other method to increase the EGFP expression cells is to increase the transfection efficiency by transduction of cells at $32^{\circ} \mathrm{C}$ and centrifugation during transduction (Bunnell et al, 1995; Movassagh et al, 2000).

Although the constructs we described can be used to analyze some B-cell processes, we propose that additional research is required to affirm the B-cell development in vivo, such as B-cell positive and negative selections in the germinal center.

In summary, membrane immunoglobulin expressed by retroviral vector gene transfer can mimic partial function of the B-cell receptor. Our system will allow investigators to assess the contribution of target molecules to B-cell signal transduction.

\section{MATERIALS AND METHODS}

\section{Luciferase assay of promoter activity}

To construct the reporter assay vectors, all DNA promoters were amplified by PCR from commercialized vectors or human genome DNA, then subcloned into pGL3-Basic vector (Promega, USA). The sequences of promoters are available in Supplemental Materials. NIH3T3 cell line (ATCC, USA) was transfected with the resulting vectors. Luciferase activity was assayed $48 \mathrm{~h}$ after transfection using a Dual-Luciferase ${ }^{\circledR}$ Reporter Assay System according to the manufacturer's instructions (Promega). Luciferase activity was normalized against transfection efficiency by using a pRL-CMV (cytomegalovirus) reporter vector (Promega) as an internal control. Results were expressed as the ratio of firefly luciferase to renilla luciferase activity.

\section{Construction and packaging of mIg-expressing retrovi- ral vector}

The EGFP (enhanced green fluorescent protein) gene was amplified by PCR from pEGFP-C1 (Clontech, USA) and subcloned into the $E c o$ R I and $B g l$ II sites of retroviral vector pMSCV-neo (Clontech), and the resulting vector was named pMEGFP. The mIg cDNA, of IgG1 isotype, was kindly donated by Professor Liang Mifang (Institute for Viral Disease Control and Prevention, Chinese Center for Disease Control and Prevention). This $\mathrm{mIg}$ is specific for the antigen of hantaan virus core protein. The mIg cDNA was subcloned into the $B g l$ II and Hind III sites of the pMEGFP vector to substitute the phosphoglycerate kinase (PGK) promoter and the Neo resistance cassette. The resulting vector, named pMEIg, contains an EGFP gene and the mIg-expressing cassette.

$18 \mathrm{~h}$ before transfection, Platinum-E cell lines were plated onto $10 \mathrm{~cm}$ plates at a density of $6 \times 10^{6}$ cells per plate in 7 mL Dulbecco's modified eagle medium (DMEM). Transfection of retroviral constructs onto Platinum-E cells was performed using Lipofectamine 2000 (Invitrogen, USA) according to the manufacturer's instructions. $24 \mathrm{~h}$ after transfection, DMED medium was replaced with $5 \mathrm{~mL}$ RPMI (Roswell Park Memorial Institute) 1640 medium (Invitrogen). Viral supernatants were collected $48 \mathrm{~h}$ after transfection and filtered $(0.45 \mu \mathrm{m})$ before used.

\section{Splenocyte separation and primary B-cell infection}

Spleens were obtained from 8-week-old BALB/c mice and placed in RPMI 1640 medium (Invitrogen). Details of the preparation of single spleen cell suspension have been described in a former report ( $\mathrm{Ru}$ and Peijie, 2009). Lymphocytes were cultured in RPMI1640 medium supplemented with $10 \%$ fetal bovine serum with $1 \mathrm{mmol} \mathrm{L}^{-1}$ sodium pyruvate, $50 \mu \mathrm{mol} \mathrm{\textrm {L } ^ { - 1 }}$ beta-mercaptoethanol, nonessential amino acid (NEAA), and $2 \mathrm{mmol} \mathrm{L}^{-1}$ Glutamine, and were stimulated with lipopolysaccharide (LPS, $50 \mu \mathrm{g} \mathrm{m}^{-1}$, Sigma-Aldrich, USA) $16 \mathrm{~h}$ before infection.

Retroviral vectors can only infect activated cells. Studies 
of the activation of murine lymphocytes by mitogens have shown that bacterial LPS activated B-cells but not Tcells (Andersson et al, 1972; Blomgren et al, 1971; Greaves et al, 1972). Gene transfer into primary B-cells was performed by cultivating splenocytes with viral supernatants in the presence of LPS $\left(50 \mu \mathrm{g} \mathrm{mL}^{-1}\right)$ and polybrene $\left(6 \mu \mathrm{g} \mathrm{mL} \mathrm{m}^{-1}\right)$. LPS-stimulated splenocytes $\left(5.0 \times 10^{7}\right)$ were plated in $10 \mathrm{~mL}$ of viral supernatants in $10 \mathrm{~cm}$ tissue culture plates, to which LPS $\left(50 \mu \mathrm{g} \mathrm{mL}^{-1}\right)$ and polybrene $(6 \mu \mathrm{g}$ $\mathrm{mL}^{-1}$ ) were also added to the culture medium. Infected B-cells were harvested $24 \mathrm{~h}$ later.

\section{Immunofluorescence detection}

Primary B-cells infected with retroviral vectors were triple-washed with ice-cold phosphate-buffered saline (PBS). Cells were then fixed with $4 \%$ paraformaldehyde solution for $20 \mathrm{~min}$ at room temperature. The cells were then washed with cold PBS and incubated with $0.5 \%$ Triton X-100 for 10 min at room temperature. $3 \%$ bovine albumin (BSA) (Sigma, USA) blocking solution was used followed by three more washes. HiLyte Fluor ${ }^{\mathrm{TM}}$ 555-conjugated (Dojindo, Japan) cognate antigen (hantaan virus core protein, kindly provided by Professor Liang Mifang, $1 \mu \mathrm{g} \mu \mathrm{L}^{-1}$ ) was incubated in the dark for $1 \mathrm{~h}$ at room temperature. The nuclei were then stained with Hoechst33258 after three washes with cold PBS. After several washes with cold PBS, cells were fixed to the glass surface with ProLong ${ }^{\circledR}$ Gold Antifade Reagent overnight and examined by a fluorescence microscope (TCS-SP2, Leica, Japan).

\section{Intracellular phosphospecific flow cytometry}

Phosflow-specific flow cytometry assay (BD Biosciences, USA) was used for intracellular phosphoprotein staining in B-cells. After infection with various retroviral vectors, splenocytes were rehydrated by $5 \mathrm{~mL}$ PBS. Cells were resuspended by vortexing and centrifugation. The cell pellet was then washed with $1 \mathrm{~mL}$ PBS with $1 \%$ BSA (Sigma), resuspended in $50 \mu \mathrm{L}$ PBS with $1 \%$ BSA, then split into new, individual flow cytometry tubes. Cognate antigen $(5 \mu \mathrm{g})$ was used for activation. PBS was used as a negative control. Cells were fixed with prewarmed Phosflow Cytofix solution (BD Biosciences) at $37^{\circ} \mathrm{C}$ for 10 min then permeabilized by adding Perm Buffer III (BD Biosciences) on ice for $30 \mathrm{~min}$. Cells were then resuspended in staining buffer (BD Biosciences) for intracellular phosphoprotein staining. Alexa Fluor 647-conjuagted anti-mouse phospho-ERK1/2 (T202/Y204), anti-mouse ZAP-70 (Y319)/Syk (Y352) or mouse $\mathrm{IgG1}$ isotypic antibody was added to different tubes and incubated at $4^{\circ} \mathrm{C}$ for $30 \mathrm{~min}$ in the dark. Cells were then washed twice with 10 volumes of staining buffer and pelleted. Samples were resuspended in $200 \mu \mathrm{L}$ staining buffer and analyzed on a FACS Calibur II cytometer (BD Biosciences).

\section{Flow cytometry to detect the expression of EGFP and mIg in B-cells}

Purified rat anti-mouse CD16/CD32 and PE rat anti-mouse CD45R/B220 were purchased from BD Biosciences. APC conjugation with antigen of hantaan virus core protein was performed using a Lightning-link Allophycocyanin-XL conjugation kit according to the manufacturer's protocols. Single cell suspensions of splenocytes infected by retroviral vector were prepared. For detection, up to $10^{6}$ cells were stained in $100 \mu \mathrm{L}$ PBS plus $1 \%$ newborn calf serum after blocking with purified anti-CD16/32 mAb. B-cells were tracked by congenic marker PE anti-mouse CD45R/B220. BCRs were detected with hantaan virus core protein conjugated to allophycocyanin. Antibody or antigen incubations were for $30 \mathrm{~min}$ at $4{ }^{\circ} \mathrm{C}$ followed by wishing once in a flow buffer (PBS+1\% newborn calf serum). Three-color flow cytometry was performed using FACS Calibur flow cytometer (BD Biosciences). The data were analyzed using CellQuest software.

\section{Detecting proliferative responses in vitro and in vivo}

Splenocytes infected by retroviral vector were cultured in RPMI1640 containing 10\% fetal bovine serum (FBS), 1

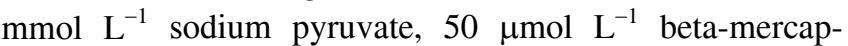
toethanol, $2 \mathrm{mmol} \mathrm{L}^{-1}$ glutamine, and NEAA, and plated into 24-well plates at a concentration of $1 \times 10^{5} \mathrm{~mL}^{-1}$ well ${ }^{-1}$ in the presence or absence of stimulators. CD40 ligand (CD40L, $100 \mathrm{ng} \mathrm{mL}^{-1}$, PerProtech, USA) and cognate antigen $(15 \mu \mathrm{g})$ were used for activation. CD40L $\left(100 \mathrm{ng} \mathrm{mL}^{-1}\right)$ and PBS were used as negative controls. After $48 \mathrm{~h}$ stimulation, $10 \mu \mathrm{mol} \mathrm{L}{ }^{-1}$ 5-ethynyl- 2'-deoxyuridine (EdU) was added to the medium for $3 \mathrm{~h}$. The proliferating cells were then measured using Click-iT EdU Alexa Fluor 647 Flow Cytometry Assay Kits (Invitrogen) according to the manufacturer's instructions. Splenocytes $\left(3 \times 10^{7}\right)$ infected by a mIg-expressing retroviral vector were transferred intravenously into SCID (severe combined immune deficiency) Beige (Vital River Laboratories, Beijing) mice. Retroviral vector pMEGFP, which encodes only EGFP were used as a negative control. After $24 \mathrm{~h}$, the mice were immunized intraperitoneally with $50 \mu \mathrm{g}$ cognate antigen or with PBS. After adoptive transfer, the splenocytes of recipients were separated and the frequency of EGFP-positive cells was detected by flow cytometry on day 18 or day 28 .

\section{Statistical analysis}

Data analysis was performed by using GraphPad Prism Statistical Software Version 5.0. Error bars indicate SDs. Dif- 
ferences among groups were determined with one-way or two-way analysis of variance (ANOVA). A value of $P<0.05$ was considered as significant.

Compliance and ethics The author( $s$ ) declare that they have no conflict of interest. All of the animal protocols were approved by the Animal Care and Use Committee at the Institute of Basic Medical Sciences, Chinese Academy of Medical Sciences and Peking Union Medical College. All efforts were made to minimize suffering.

Acknowledgements This work was supported by the National Basic Research Program of China (2011CB965203) to Yue Huang.

Andersson, J., Moller, G., and Sjoberg, O. (1972). Selective induction of DNA synthesis in T and B lymphocytes. Cell Immunol 4, 381-393.

Blomgren, H., and Svedmyr, E. (1971). Evidence for thymic dependence of PHA-reactive cells in spleen and lymph nodes and independence in bone marrow. J Immunol 106, 835-845.

Bunnell, B.A., Muul, L.M., Donahue, R.E., Blaese, R.M., and Morgan, R.A. (1995). High-efficiency retroviral-mediated gene transfer into human and nonhuman primate peripheral blood lymphocytes. Proc Natl Acad Sci USA 92, 7739-7743.

Flaswinkel, H., and Reth, M. (1994). Dual role of the tyrosine activation motif of the Ig-alpha protein during signal transduction via the B cell antigen receptor. EMBO 13, 83-89.

Glimcher, L.H., Kim, K.J., Green, I., and Paul, W.E. (1982). Ia antigen-bearing $\mathrm{B}$ cell tumor lines can present protein antigen and alloantigen in a major histocompatibility complex-restricted fashion to antigen-reactive T cells. J Exp Med 155, 445-459.

Greaves, M., and Janossy, G.. (1972). Elicitation of selective T and B lymphocyte responses by cell surface binding ligands. Transplant Rev $11,87-130$.

Justemen, L.B., Campbell, K.S., Chien, N.C., and Cambier, J.C. (1991). Regulation of B cell antigen receptor signal transduction and phosphor ylation by CD45. Science 252, 1839-1842.

Khamlichi, A.A., Glaudet, F., Oruc, Z., and Le Bert, M. (2004). Immunoglobulin class-switch recombination in mice devoid of any $\mathrm{S}$ mu tandem repeat. Blood 103, 3828-3836.

Kurosaki, T. (2011). Regulation of BCR signaling. Mol Immunol 48, 1287-1291.

Luby, T.M., Schrader, C.E., Stavnezer, J., and Selsing, E. (2001). The mu switch region tandem repeats are important, but not required, for antibody class switch recombination. J Exp Med 193, 159-168.

Movassagh, M., Boyer, O., Burland, M.C., Leclercq, V., Klatzmann, D., and Lemoine, F.M. (2000). Retrovirus-mediated gene transfer into T cells: $95 \%$ transduction efficiency without further in vitro selection. Hum Gene Ther 11, 1189-1200.

Niiro, H., and Clark, E.A. (2002). Regulation of B-cell fate by antigen-receptor signals. Nat Rev Immunol 2, 945-956.

Overell, R.W., Weisser, K.E., and Cosman, D. (1988). Stably transmitted triple-promoter retroviral vectors and their use in transformation of primary mammalian cells. Mol Cell Biol 8, 1803-1808.

Reth, M., and Wienands, J. (1997). Initiation and processing of signals from the B-cell antigen receptor. Annu Rev Immunol 15, 453-479.

Ru, W., and Peijie, C. (2009). Modulation of NKT cells and Th1/Th2 imbalance after alpha-GalCer treatment in progressive load-trained rats. Int J Biol Sci 5, 338-343.

Sanchez, M., Misulovin, Z., Burkhardt, A.L., Mahajan, S., Costa, T., Franke, R., Bolen, J.B., and Nussenzweig, M. (1993). Signal transduction by immunoglobulin is mediated through Ig alpha and Ig beta. J Exp Med 178, 1049-1055.

Schrader, C.E., Guikema, J.E., Linehan, E.K., Selsing, E., and Stavnezer, J. (2007). Activation-induced cytidinedeaminase- dependent DNA breaks in class switch recombination occur during G1 phase of the cell cycle and depend upon mismatch repair. J Immunol 179, 6064-6071.

Szymczak, A.L., Workman, C.J., Wang, Y., Vignali, K.M., and Dilioglou, S. (2004). Correction of multi-gene deficiency in vivo using a single 'self-cleaving' 2A peptide-based retroviral vector. Nat Biotechnol 22, 589-594.

Szymczak-Workman, A.L., Vignali, K.M., and Vignali, D.A. (2012). Design and construction of $2 \mathrm{~A}$ peptide-linked multicistronic vectors. Cold Spring Harb Protoc 2, 199-204.

Open Access This article is distributed under the terms of the Creative Commons Attribution License which permits any use, distribution, and reproduction in any medium, provided the original author(s) and source are credited.

\section{SUPPORTING INFORMATION}

\section{S1 Sequences of eight promoters}

The supporting information is available online at life.scichina.com and link.springer.com. The supporting materials are published as submitted, without typesetting or editing. The responsibility for scientific accuracy and content remains entirely with the authors. 